\title{
REVIEW
}

\section{Colony-stimulating factors as an adjunct to chemotherapy in small cell lung cancer}

\author{
T. Urban, M-P. Schuller, B. Lebeau
}

Colony-stimulating factors as an adjunct to chemotherapy in small cell lung cancer. T. Urban, M-P. Schuller, B. Lebeau. @ERS Journals 1996.

ABSTRACT: Myelosuppression is the major dose-limiting toxicity of chemotherapy in small cell lung cancer (SCLC). The capacity of colony stimulating factors (CSFs) to stimulate granular neutrophil recovery may be of great value to prevent or cure febrile neutropenia and to increase dose-intensity. The aim of this review was to assess the current use of CSFs in SCLC on the basis of experimental and clinical data.

Primary CSF administration has been shown to reduce the incidence of febrile neutropenia, hospital admission rate, and antibiotic use subsequent to cyclophosphamidedoxorubicin-high dose etoposide (CDE) chemotherapy, without improvement of survival or disease control. Primary CSF administration may be recommended when the expected incidence of febrile neutropenia is at least $40 \%$. This benefit has not been established with less myelosuppressive regimens, such as cisplatin-etoposide (PE), which remains an alternative combination in SCLC when standard doses are used. A trial comparing high-dose CDE + CSF with PE would be of considerable interest.

There is currently little clinical basis for the use of CSFs to increase chemotherapy dose-intensity, outside clinical trials. Peripheral blood progenitor cells mobilized with CSFs offer interesting prospects. Further studies, with later initiation, shorter duration or lower doses of CSFs, are warranted to improve the cost-effectiveness of CSFs.

CSF therapy in addition to antibiotics is normally not justified in febrile neutropenia, except perhaps in selected patients with sepsis syndromes, hypotension or pneumonia.

Eur Respir J., 1996, 9, 596-602
Service de Pneumologie, Hôpital SaintAntoine, Paris France.

Correspondence: T. Urban

Service de Pneumologie

Hôpital Saint-Antoine

184 rue du Faubourg Saint-Antoine Paris 75012

France

Keywords: Chemotherapy

colony-stimulating factor

dose-intensity

febrile neutropenia

progenitors

small cell lung cancer

Received: April 191995

Accepted after revision November 201995
The objective of lung cancer treatment is the best beneficial effect on length and quality of life with good costeffectiveness, and the lowest morbidity and mortality. In current practice, neutropenia, and sometimes thrombocytopenia are the major dose-limiting toxicities of most chemotherapy regimens, particularly in small cell lung cancer (SCLC). Their consequences are potentially life-threatening infections and, more rarely, haemorrhage, dose reduction, delayed chemotherapy, and deterioration of quality of life. Because fever is often the only manifestation of infection, broad-spectrum antibiotic therapy is usually initiated early.

The in vitro and in vivo experimental capacities of granulocyte colony-stimulating factors (G-CSFs) and granulocyte-monocyte colony-stimulating factors (GMCSFs) to stimulate granular neutrophil recovery may be of great value to prevent or cure febrile neutropenia induced by chemotherapy. CSFs could, therefore, have a valuable role in improving the acceptability of chemotherapy by reducing clinical infections, admission to hospital for haematological toxicity, and the need for antibiotic therapy. Another goal of CSFs would be to improve tumour responses to chemotherapy and survival by increasing the dose-intensity of chemotherapy. This survival improvement has not yet been achieved, partly because of the limitation of chemotherapy by nonmyeloid toxicities when high dosages are used. Because of the high cost and many potential indications of CSFs, socioeconomic evaluation of these agents is necessary.

The aim of this review is to assess the current use of CSFs in SCLC on the basis of clinical data and our own experience. A computerised literature search for trials, reviews, and official guidelines (American Society of Clinical Oncology (ASCO), European Society of Medical Oncology (ESMO)) (Medline) has been performed. Quality criteria were applied for selection and interpretation of the studies, such as randomization, statistical power, and number of patients.

\section{Haematological effects of colony-stimulating factors}

Specific colony-stimulating factors regulate the production of various mature peripheral blood cells. Monocytes and neutrophils are derived from common progenitors called granulocyte and monocyte colony forming units (GM-CFU). GM-CSF stimulates the production of neutrophil granulocytes, monocytes and macrophages, G-CSF stimulates the 
production of neutrophil granulocyte production, and macrophage colony-stimulating factor (M-CSF) stimulates monocyte and macrophage production.

Two recombinant G-CSFs are currently marketed in Europe: filgrastim or r-met-hu-G-CSF (Neupogen®, Amgen), which is the most extensively studied variety, and, more recently, lenograstim or r-hu-G-CSF, a glycosylated GCSF (Granocyte ${ }^{\circledR}$, Bellon). GM-CSF or molgramostim (Leucomax $®$, Schering Plough) has been less extensively studied in SCLC therapy than in haematological disorders. Clinical studies with interleukin-3 (IL-3), particularly for its platelet action, and M-CSF are still ongoing.

The biological effects of G-CSF on neutrophils consist of three consecutive phases [1-5]. The first phase is a rapid and brief moderate fall in neutrophil count, within minutes after intravenous injection, due to margination. The second phase corresponds to a rise in neutrophil count within $24 \mathrm{~h}$ due to release of preformed granulocytes located in the bone marrow and accelerated survival of blood neutrophils. The third phase consists of an increase in circulating functional neutrophils due to amplification in the bone marrow maturation compartment. Mature neutrophils are more rapidly mobilized into the circulation.

Treatment with G-CSF leads to a marked redistribution of haematopoiesis $[6,7]$, without depletion of stem/ progenitor cell populations [8]. In animal models, neutrophils produced after G-CSF treatment are able to control infections due to a range of different bacteria [9]. Neutrophils harvested from patients in vivo are functional (phagocytosis, superoxide generation, chemotaxis), with enhanced functions [10-12]. The effects of CSFs on neutrophils are dose-dependent [6], and long-term administration of GCSF is possible [13]. GM-CSF administration results in a similar but slower stimulation of neutrophils, as well as an action on monocyte and eosinophil counts.

CSFs are usually well-tolerated. The most serious adverse effect of filgrastim is moderate medullary bone pain in $10-39 \%$ of cases, compared to $0-21 \%$ of cases following placebo. Subcutaneous injection site reactions are rare. Moderate thrombocytopenia has been observed in filgrastim randomized studies, but was not associated with haemorrhage [10, 14-16]. Anti-G-CSF antibodies have not yet been reported [17-19]. Other adverse effects are very infrequent. Various adverse effects after GM-CSF therapy include fever, injection site reaction, asthenia, myalgia, bone pain, nausea and, rarely, rash, pruritus, oedema, paraesthesia, anorexia and diarrhoea [20]. Flushing with tachycardia, hypotension, and dyspnoea has been noted after the first injection of GM-CSF, particularly molmograstim, in SCLC patients [21-23]. Fever seems more frequent with GM-CSF.

CSFs should be used cautiously in lung cancer patients receiving concomitant chemotherapy and radiation therapy, according to two studies of patients with GM-CSF in SCLC and G-CSF in non-small cell lung cancer. In both studies, thrombocytopenia was significantly more severe in the CSF group than in the placebo group [24, 25]. Nevertheless, thrombocytopenia has been reported in most studies, using both G- and GM-CSF, regardless of the use of concomitant radiotherapy. This effect could simply reflect the increased chemotherapy dose-intensity in CSFtreated patients. The issue is still controversial and requires further evaluation.

\section{Primary CSF administration}

This strategy is defined as the administration of CSFs before the onset of neutropenia or febrile neutropenia. The management of SCLC with chemotherapy leads to a four- or fivefold increase in median survival. Three year survival may be observed in $5-10 \%$ of patients, depending on the inclusion criteria. In large scale studies, $2-4 \%$ of patients achieved five-year survival [26, 27]. Minor improvement in median survival has been observed when thoracic radiotherapy is added to chemotherapy in limited disease [28, 29], or with subcutaneous heparin treatment [30]. No major additional improvement has recently been achieved.

Neutropenia and subsequent infections are the major causes of morbidity and toxic mortality in SCLC patients treated with chemotherapy. Septic deaths during febrile neutropenia may be observed in about $5 \%$ of patients, and sometimes $8 \%$ with high-dose chemotherapy [31]. Grade and duration of neutropenia have been correlated to the risk of developing sepsis [32]. In addition to the impact on the rate of hospitalization, and the need for intravenous antibiotic therapy, febrile neutropenia results in delays of subsequent chemotherapy and dose reductions. Disease progression, performance status, and age are predictive factors for severe neutropenia and toxic death [33, 34]. Broad-spectrum antibiotic therapy is currently initiated immediately in patients with febrile neutropenia at the onset of fever, usually with good results in terms of mortality [35]. According to a recent multivariate analysis, neutrophil recovery was a favourable prognostic factor in such situations, independently of the use of antibiotic therapy [36, 37]. CSFs could, therefore, play an interesting role in improving the acceptability of chemotherapy by reducing clinical infections, admissions to hospital for haematological toxicity, and the need for antibiotic therapy. CSFs could also allow increasing dose-intensity of chemotherapy.

\section{CSF effects on haematological toxicity with chemotherapy}

The most widely-used combination chemotherapy regimens are cyclophosphamide-doxorubicin-etoposide (CDE), cyclophosphamide-doxorubicin (Adriamycin)-vincristine (CAV) and cisplatin-etoposide (PE), with a fairly comparable efficacy, particularly for CAV and PE [38, 39]. A lower incidence of myelosuppression has usually been observed with the PE regimen, which appears preferable to $\mathrm{CDE}$ or CAV regimens when standard doses are used. The CDE combination chemotherapy used in most of the following studies was administered every 3 weeks, with high-dose etoposide [17, 18].

\section{$G-C S F S$}

Bronchud et al. [8] evaluated the action of G-CSF in a phase I/II study and demonstrated an increased neutrophil count, with normal neutrophil function tests of mobility and bactericidal activity.

G-CSF has been studied in two large multicentre, randomized phase III trials in the US and Europe. Patients 
were treated with CDE chemotherapy every 3 weeks $[17$, 18]. The principal difference between the US [17] and European [18] studies was that, in the US trial, patients found to be receiving placebo on unblinding were crossed over to open label G-CSF for the remaining cycles.

The US placebo-controlled trial, published in 1991, included 211 patients with SCLC [17]. The patients were treated with CDE chemotherapy combined with either subcutaneous filgrastim, at a daily dose of $230 \mu \mathrm{g} \cdot \mathrm{m}^{-2}$ for up to 14 days, or placebo. Febrile neutropenia was defined as an absolute neutrophil count less than $0.5 \times 10^{9} \cdot \mathrm{L}^{-1}$ with axillary fever $>38.2^{\circ} \mathrm{C}$. A significant reduction of the incidence of febrile neutropenia was observed in the filgrastim group, in the first cycle of chemotherapy from 57 to $28 \%(p<0.001)$, and for all cycles from 77 to $40 \%$. Duration of febrile neutropenia, rate and duration of hospitalization for infection (first cycle), and antibiotic use (first cycle) were also significantly reduced in the filgrastim group. Bacteriological cultures confirmed a lower incidence of infection in the G-CSF group (6.5\%) than in the placebo group (13.3\%). No data are available concerning the effect of G-CSF on dose-intensity, treatment outcome, and quality of life [17].

In the European multicentre placebo-controlled trial, published in 1993 [18], in which we participated, 130 SCLC patients were treated with CDE and either filgrastim $\left(230 \mu \mathrm{g} \cdot \mathrm{m}^{-2}\right.$ daily, on days $\left.4-17\right)$ or placebo. Febrile neutropenia was defined as an absolute neutrophil count less than $1.0 \times 10^{9} \cdot \mathrm{L}^{-1}$ with axillary fever $>38.2^{\circ} \mathrm{C}$. This study demonstrated a significant reduction in febrile neutropenia from 41 to $20 \%(\mathrm{p}<0.001)$ during the first cycle. The cumulative febrile neutropenia rate was $53 \%$ in the placebo group and $26 \%$ in the G-CSF group $(\mathrm{p}<0.002)$. This beneficial effect of G-CSF in terms of haematological toxicity was mainly observed during the first cycle. Few patients without febrile neutropenia during the first cycle subsequently developed febrile neutropenia, whether or not they were receiving G-CSF. Filgrastim also reduced the hospital admission rate $(\mathrm{p}<0.04)$, and antibiotic use $(p<0.02)$. These effects were maintained throughout six cycles of chemotherapy. Furthermore, filgrastim compared with placebo, significantly decreased the need for chemotherapy dose reduction $(\mathrm{p}<0.001)$ and the incidence of delayed chemotherapy $(\mathrm{p}<0.04)$. Thrombocytopenia was significantly more marked after the sixth CDE cycle in the filgrastim group than in the placebo group, without any clinical consequences. The reason may be the higher doses of chemotherapy given in the G-CSF group, or GCSF toxicity [18]. Data concerning the effect of GCSF on quality of life are not available [18].

These two studies used febrile neutropenia as the major end-point, rather than the more important end-point of the incidence of documented bacterial and/or fungal infections $[17,18]$. One explanation for this choice is the great difficulty of proving such infection during febrile aplasia of solid tumours, which is shorter than chemotherapy-induced aplasia in haematological malignancies. No difference was observed in the toxic mortality rate between G-CSF and placebo in either study, partly due to the small sample size, the efficacy of antibiotics, and the relatively short duration of febrile neutropenia.
In another smaller randomized trial, 40 patients with SCLC were given weekly courses of cisplatin-etoposide alternating with ifosfamide-doxorubicin, with or without G-CSF. No reduction in febrile neutropenia and septic mortality was reported, despite less severe neutropenia in the G-CSF group [40]. The small sample size of the study could account for the absence of any intergroup difference.

\section{GM-CSFS}

GM-CSF preventive treatment with standard-dose chemotherapy has been less consistently beneficial in SCLC. A randomized study using molgramostim (Leucomax ${ }^{\circledR}$ ) versus placebo after CDE chemotherapy in 148 patients with SCLC resulted in faster neutrophil recovery, less severe neutropenia, and reduced antibiotic use during the first cycle of chemotherapy, but with no significant effect on the incidence of febrile-neutropenia and chemotherapy dose-intensity [41].

Another larger study in 230 patients with limited-stage SCLC receiving $\mathrm{PE}$ and simultaneous chest radiation, with or without GM-CSF (sargramostim), failed to demonstrate any significant benefit with GM-CSF. There was a statistically significant increase in the frequency and duration of life-threatening thrombocytopenia in patients randomized to GM-CSF. GM-CSF patients had significantly more toxic deaths, more nonhaematological toxicities, more days in hospital, and a higher incidence of intravenous antibiotic use [25]. GM-CSF patients had higher neutrophil nadirs $(\mathrm{p}<0.01)$, but no significant difference in the frequency of grade 4 neutropenia. There were no significant difference in survival. The low frequency of severe haematological toxicity with the PE regimen obviates the need for CSF use.

\section{CSFs effects on median survival time}

No significant difference in median survival time was observed between the two groups in the US and European trials $[17,18]$. Overall response rates were not significantly different between the filgrastim and placebo groups, despite better compliance with CDE dose-intensity in the filgrastim group in the European trial [18]. A larger study population would be required to detect the small expected survival benefit with G-CSF.

One explanation could be the similar dosage of CDE chemotherapy for the first cycle in the two groups of patients, since it has been demonstrated that doctor's compliance with the initial chemotherapy dose is an important prognostic factor in multivariate analysis [42]. Absolute compliance with subsequent chemotherapy dosage is perhaps less important than initial compliance during the first cycle. It would, therefore, be interesting to evaluate the benefit of an increased initial dosage of chemotherapy right from the first cycle with G-CSF.

Despite the initial chemosensitivity of SCLC, the presence of chemoresistant tumour clones at the time of 
diagnosis in the two groups may be another explanation for the similar failure rate of chemotherapy. Nevertheless, a high initial toxic effect on tumour cells could probably limit the emergence of acquired resistance.

Another explanation may be the hypothetical role of G-CSF as a growth factor for tumour cells, supported by an $\mathrm{M}-\mathrm{CSF}$, and G-CSF in vitro induction of cell replication in an SCLC cell line (NCI 417) [43]. In contrast, an in vitro antiproliferative and differentiation effect of GM-CSF was demonstrated on an SCLC line [44]. More recent data have not shown significant in vitro growth stimulation for either G-CSF or GM-CSF [45, 46]. The issue remains controversial.

\section{Economic studies}

Cost-benefit analysis of CSFs should be performed because they are very expensive. Such procedures are not easy. Reduction of febrile neutropenia and hospitalization should be taken into account. As economic studies have not been prospectively incorporated into CSF clinical trials, an accurate assessment of economic consequences cannot be made. The only prospective economic data available are related to the number of days in hospital (one of the major cost determinants), the use of antibiotic therapy, and sometimes the use of blood transfusions.

NichOLs et al. [47] retrospectively reviewed records of 137 consecutive patients with SCLC treated with standard-dose combination chemotherapy. In this study, the incidence of neutropenic fever was only $18 \%$, to be compared with the placebo- and G-CSF-treated arms of the US trial [17], in which the incidence of neutropenic fever was 77 and 40\%, respectively. This study suggested a better cost-benefit when G-CSF was given only to patients who developed an infection during a previous chemotherapy cycle. According to these data, secondary use of CSFs could be proposed with an economic objective, as it avoids the use of CSFs in patients who might not need them. Moreover, the US study suggested that patients with a previous history of febrile neutropenia are at higher risk for recurrent fever [17].

In fact, toxic deaths during febrile neutropenia are significantly more frequent in the first cycle of chemotherapy in SCLC than in subsequent courses [33], and the risk of sepsis is greater during the first cycle of chemotherapy in patients with a large tumour burden [42]. It can be hypothesized that the first cycle decreases the likelihood of infection during subsequent cycles by reducing tumour bulk. Consequently, a large part of the benefit of primary CSF prophylaxis would be lost with secondary CSF administration. CSFs should, therefore, be used as preventive treatment right from the first cycle when heavily myelosuppressive chemotherapy is used, such as highdose CDE or cisplatin-CDE (PCDE). Secondary CSF administration could be evaluated with less toxic chemotherapy, such as cisplatin and etoposide, in case of febrile neutropenia, to avoid a reduction of dose-intensity of the subsequent courses. Another economic strategy, which remains to be evaluated, is the preventive administration of CSF to all patients in the first cycle, and for subsequent cycles only to those patients who developed febrile neutropenia.

\section{Dosage and duration of CSF treatment}

In adults, the recommended CSF doses are $5 \mu \mathrm{g} \cdot \mathrm{kg}^{-1}$ daily of G-CSF (filgrastim) or $250 \mu \mathrm{g} \cdot \mathrm{m}^{-2}$ daily of GMCSF (sargramostim). Clinical data suggest that starting G-CSF or GM-CSF within 24-72 h after chemotherapy and continuing treatment beyond the leucocyte nadir until the onset of an absolute neutrophil count of $10 \times$ $10^{9}$ cells $\cdot \mathrm{L}^{-1}$ may ensure effective neutrophil recovery. For example, G-CSF, at a dosage of $230 \mu \mathrm{g} \cdot \mathrm{m}^{-2}$, was shown to be effective in reducing the incidence of fever and neutropenia [17-19]. However, certain data suggest that G-CSF doses as low as $50 \mu \mathrm{g} \cdot \mathrm{m}^{-2}$ may be effective for primary G-CSF administration after chemotherapy, whilst reducing cost and avoiding waste [48-49]. Further studies are required to determine the optimal CSF doses to achieve clinical benefits.

Cost reduction of primary preventive G-CSF therapy, with later administration or shorter duration of administration sufficient to achieve a neutrophil count of up to $1.0 \times 10^{9}$ cells $\cdot \mathrm{L}^{-1}$ with clinical recovery, should also be considered. Ongoing clinical studies are being conducted to investigate this issue. We are currently conducting a randomized phase II trial comparing the effects of later administration of G-CSF at Day 4 or 7 subsequent to chemotherapy in SCLC patients.

\section{High dose-intensity chemotherapy and CSFs}

One of the major hopes raised by CSFs is related to their theoretical ability to allow more intensive chemotherapy and, consequently, to improve dose-intensity, response rate, and survival.

\section{Intensification of chemotherapy with CSFS}

The superiority of 3-4 drug combinations over 2 drug combinations with platinum is based on theoretical considerations as well as some clinical studies [50]. However, other studies had not demonstrated any survival difference between 2, 3 or 4 drugs, partly because of toxic deaths with 4 drug chemotherapy [39].

A randomized study in 399 SCLC patients receiving CDE versus PCDE chemotherapy, without CSF, failed to demonstrate any significant survival benefit despite a higher response rate in the PCDE group $(\mathrm{p}<0.001)$, due to more pronounced haematological toxicity in this group $(p<0.001)$ [31]. The use of G-GSF as an adjunct to PCDE chemotherapy would probably reduce neutropenia, but thrombocytopenia remains a major limitation of this intensive chemotherapy [51].

Another strategy for increasing dose-intensity chemotherapy is the use of initial or late intensive high doses of the same drugs [42]. Several studies have failed to demonstrate any major improvement in median survival [52-54]. However, a recent study in limited SCLC demonstrated that a $20 \%$ higher initial dosage of cyclophosphamide and cisplatin achieved a $25 \%$ increase in 
complete remission rate, a $50 \%$ increase in the duration of complete remission and a better 2 year survival rate $(p=0.016)$ [42]. In the European study, an increase of $8 \%$ of CDE dose-intensity was reported with G-CSF, without any survival improvement [18]. WoLL et al. [55] reported a modest but significant increase in dose-intensity with decreasing treatment interval of a 4 drug chemotherapy (vincristine, ifosfamide, carboplatin and etoposide) with G-CSF (lenograstim) compared with control (25 versus $18 \%$; $\mathrm{p}=0.03$ ). The increase in cytotoxic dose-intensity in the G-CSF group was associated with a better 2 year survival rate (32 versus 15\%) [55]. HАмм et al. [41] also demonstrated a higher dose-intensity for GM-CSF treated patients with the CDE regimen [41]. Others phase II studies have suggested the possibility of increasing dosage of chemotherapy by $20-40 \%$ with CSF support [56-58], but survival data are not available.

The choice of agents such as doxorubicin and cisplatin can be questioned. Severe extramedullary toxicity (skin mucositis) was observed when doxorubicin was doseintensified with G-CSF. Cisplatin with its potential for nephro- and neurotoxicity is also an unlikely candidate for dose intensification.

\section{CSFs and peripheral blood progenitor cells}

Intensification of chemotherapy, with repeated administration of CSF mature peripheral blood progenitor cells (PBPC) offers interesting prospects in SCLC. The capacity of CSFs to mobilize haematopoietic stem cells and progenitor cells is being evaluated as replacement therapy for bone marrow transplantation. PBPC are collected by leukapheresis or venosection after stimulation with G-CSF or GM-CSF at each cycle of chemotherapy, and reinfused on Day 3 of the next cycle in order to accelerate haematopoietic recovery $[59,60]$. Reverse transcriptase-polymerase chain reaction (RT-PCR) studies for detection of residual disease have shown that tumour contamination was more common than previously thought [61]. Pettengell et al. [62] recently demonstrated that ifosfamide, carboplatin and etoposide chemotherapy with G-CSF was effective in mobilizing PBPC, without evidence of stem cell depletion in long-term cultures. The dose intensity can be doubled by reinfusing haematopoietic progenitors. No survival data are yet available.

\section{CSF therapy}

CSFs may be used as an adjunct to antibiotic therapy. This strategy is proposed with an economic goal, because it avoids the use of CSFs in patients who might not need them. No such study has yet been conducted specifically in SCLC patients. In the US, $14 \%$ of filgrastim indications correspond to treatment of episodes of postchemotherapy febrile neutropenia after they occur rather than to prevent them (unpublished market research data, Amgen, Inc., [63]). Five placebo-controlled randomized studies have evaluated G-CSF and GM-CSF as adjuncts to antibiotics [63-66].
MAHER et al. [63] reported a randomized, double-blind, placebo-controlled study of G-CSF therapy with various chemotherapy regimens in 218 patients with cancer other than myeloid leukaemia. In this study, patients with fever greater than $38^{\circ} \mathrm{C}$ and neutrophils less than $10 \times$ $10^{9}$ cells $\cdot \mathrm{L}^{-1}$ were treated with piperacillin-tobramycin and with either high-dose filgrastim $\left(12 \mu \mathrm{g} \cdot \mathrm{kg}^{-1}\right.$ daily) or placebo. This treatment was given until neutrophil count exceeded $0.5 \times 10^{9}$ cells $\cdot L^{-1}$, with 4 days without fever [63]. These authors found a significant but moderate reduction of duration of neutropenia ( 3 versus 4.4 days; $\mathrm{p}=0.005$ ), and resolution of febrile neutropenia ( 5 versus 6 days; $\mathrm{p}=0.01$ ) compared to placebo. This benefit was higher in patients with documented infections, with neutrophil count less than $0.5 \times 10^{9}$ cells $\cdot \mathrm{L}^{-1}$, and with solid tumours $(\mathrm{p}<0.001)$. No difference was observed with respect to duration of fever, platelet transfusion requirements and death rate. Similar clinical findings have been reported with molgramostim, with a possible benefit for patients with clinical tissue infections [64].

In contrast, other studies have reported more favourable results, with a reduction of hospital stay $[65,66]$. Similar toxic mortality rates were observed in these studies $(2 \%$ in the placebo groups versus $4 \%$ in the CSF groups) [63-66]. CSF therapy requires additional evaluation, particularly in high-risk patients with pneumonia, sepsis syndrome, hypotension or tissue infection.

In conclusion, primary CSF administration is recommended in patients with SCLC treated with high-dose CDE chemotherapy, or when the expected incidence of febrile neutropenia with chemotherapy is greater than or equal to $40 \%$. The value of primary administration of CSFs has not been established in less myelosuppressive regimens, such as PE. The absence of beneficial effect on median survival in the high dosage CDE-CSF group in randomized studies would justify a trial comparing this regimen versus PE chemotherapy in order to demonstrate a benefit of high dose CDE-CSF.

There is currently little basis for the use of CSFs to increase chemotherapy dose-intensity, outside of clinical trials. PBPC mobilized with CSFs offers interesting prospects in solid tumours. Cost reduction of primary CSF administration, with later initiation, shorter duration, or lower doses of CSFs, sufficient to achieve a neutrophil count of up to $1.0 \times 10^{9}$.cells $\cdot \mathrm{L}^{-1}$ with clinical recovery, must also be considered in future studies.

Secondary CSF administration may be an alternative to the use of dose reduction to decrease the probability of febrile neutropenia in subsequent cycles of chemotherapy.

Clinical trial data do not clearly justify the use of CSF therapy in addition to antibiotic therapy in febrile neutropenia. However, selected patients with bacterial or fungal sepsis syndromes, hypotension, or pneumonia with poor prognostic factors could benefit from CSF therapy.

\section{References}

1. Platzer E. Human haematopoietic growth factors. Eur J Haematol 1989; 42: 1-15.

2. Lieschke GJ, Burgess AW. Granulocyte colony-stimulating 
factor and granulocyte macrophage colony-stimulating factor. N Engl J Med 1992; 327: 28-35.

3. Lieschke GJ, Burgess AW. Granulocyte colony-stimulating factor and granulocyte macrophage colony-stimulating factor. N Engl J Med 1992; 327: 99-106.

4. Lord BI, Bronchud MH, Owens S, et al. The kinetics of human granulopoiesis following treatment with granulocyte colony-stimulating factor in vivo. Proc Natl Acad Sci USA 1989; 86: 9499-9503.

5. Lord BI, Gurney H, Chang J, et al. Haematopoietic cell kinetics in humans treated with rGM-CSF. Int J Cancer 1992; 50: 26-31.

6. Molineux G, Dexter TM. 1. Biology of G-CSF. In: Morstyn G, Dexter TM, eds. Filgrastim -r-met-huG-CSF in Clinical Practice. Basic \& Clinical Oncology Series. New York, Marcel Dekker, 1993; Vol. 5, pp. 1-21.

7. Tamura $\mathrm{M}$, Hattori $\mathrm{K}$, Nomura $\mathrm{H}$, et al. Induction of neutrophilic granulocytosis in mice by administration of purified human native granulocyte colony-stimulating factor (G-CSF). Biochem Biophys Res Commun 1987; 142: 454-460.

8. Bronchud MH, Scarffe JH, Thatcher N, et al. Phase I/II study of recombinant human granulocyte colony-stimulating factor in patients receiving intensive chemotherapy for small cell lung cancer. Br J Cancer 1987; 56: 809-813.

9. Matsumoto M, Matsubara S, Matssuno M, et al. Protective effect of human granulocyte colony-stimulating factor on microbial infection in neutropenic mice. Infect Immunol 1987; 55: 2715-2720.

10. Lindemann A, Herrmann F, Oster W, et al. Hematologic effects of recombinant human granocyte colony-stimulating factor in patients with malignancy. Blood 1989; 74: 2644-2651.

11. Bronchud MH, Potter MR, Morgenstern G, et al. In vitro and in vivo analysis of the effects of recombinant human granulocyte colony-stimulating factor in patients. Br J Cancer 1988; 58s: 64-69.

12. Ohsaka A, Kitagawa $\mathrm{S}$, Sakamoto $\mathrm{S}$, et al. In vivo activation of human neutrophil functions by administration of recombinant human granulocyte colony-stimulating factor in patients with malignant lymphoma. Blood 1989; 74: 2743-2748.

13. Chang JM, Metcalf D, Gonda TJ, Johnson GR. Longterm exposure to retrovirally expressed granulocyte colonystimulating factor induces a non-neoplastic granulocytic and progenitor cell hyperplasia without tissue damage in mice. J Clin Invest 1989; 84: 1488-1496.

14. Bonnilla MA. The Severe Chronic Neutropenia Study Group in conjunction with Amgen Inc. Clinical efficacy of recombinant human granulocyte colony-stimulating factor (r-met-Hu-g-CSF) in patients with severe chronic neutropenia. Blood 1990; 76: 133a.

15. Mueller BU, Jacobsen F, Butler KM, et al. Combination treatment with azidothymidine and granulocyte colonystimulating factor in children with human immunodeficiency virus infection. J Pediatr 1992; 121: 797-802.

16. Sandoval C, White M, Jennings L, et al. Platelet dysfunction in patients with chronic neutropenia treated with G-CSF. Blood 1992; 80: 502a

17. Crawford J, Ozer H, Stoller R, et al. Reduction by granulocyte colony stimulating factor of fever and neutropenia induced by chemotherapy in patients with small-cell lung cancer. N Engl J Med 1991; 325: 164-170.

18. Trillet-Lenoir V, Green J, Manegold C, et al. Recombinant granulocyte colony-stimulating factor reduces the infectious complications of cytotoxic chemotherapy. Eur J Cancer 1993; 29: 319-324.
19. Pettengell R, Gurney H, Radford JA, et al. Granulocyte colony-stimulating factor to prevent dose-limiting neutropenia in non Hodgkin's lymphoma: a randomised controlled trial. Blood 1992; 80: 1430-1436.

20. American Society of Clinical Oncology. Recommendations for the use of hematopoietic colony-stimulating factors: evidence-based, clinical practice guidelines. J Clin Oncol 1994; 12: 2471-2508.

21. Vadhan-Raj S, Keating M, LeMaistre A, et al. Effects of recombinant human granulocyte-macrophage colonystimulating factor in patients with myelodysplastic syndromes. N Engl J Med 1987; 317: 1445-1452.

22. Lieschke GJ, Morstyn G. Role of G-CSF and GM-CSF in the prevention of chemotherapy-induced neutropenia. In: Meltelsmann $\mathrm{R}$, Herrmann $\mathrm{F}$, eds. Hematopoietic Growth Factors in Clinical Applications. Dekker, NewYork, 1992; pp. 191-223.

23. Rusthoven J, Goss P, Eisenhauer E. Phase I-II trial of high dose cisplatin, etoposide, and GM-CSF for small cell lung cancer (SCLC). Proc Am Soc Clin Oncol 1991; 10: 251.

24. Momin F, Kraut M, Lattin P, et al. Thrombocytopenia in patients receiving chemoradiotherapy and G-CSF for locally advanced non-small cell lung cancer (NSCLC). Proc Am Soc Clin Oncol 1992; 11: 294.

25. Bunn PA, Crowley J, Kelly K, et al. Chemoradiotherapy with or without granulocyte-macrophage colony-stimulating factor in the treatment of limited-stage small cell lung cancer: a prospective phase III randomized study of the South-west Oncology Group. J Clin Oncol 1995; 13: 1632-1641.

26. Souhami RL, Law K. Longevity in small cell lung cancer: a report to the Lung Cancer Subcommittee of the United Kingdom Coordinating Committee for Cancer Research. Br J Cancer 1990; 61: 584-589.

27. Rosti G, Donadio M, Crino L, et al. Long survivors in small cell lung cancer (SCLC): Italian report on 3,245 cases. Proc Am Soc Clin Oncol 1991; 10: 268.

28. Pignon JP, Arriagada R, Idhe DC, et al. A meta-analysis of thoracic radiotherapy for small cell lung cancer. N Engl J Med 1992; 327: 1618-1624.

29. Murray N, Coy P, Pater JL, et al. Importance of timing for thoracic irradiation in the combined modality treatment of limited-stage small cell lung cancer. J Clin Oncol 1993; 11: 336-344.

30. Lebeau B, Chastang CI, Bréchot JM, et al. Subcutaneous heparin treatment increases survival in small cell lung cancer. Cancer 1994; 74: 38-45.

31. Lebeau B, Chastang CI, Urban T, et al. Cytoxandoxorubicin-etoposide (CDE) vs cisplatin - CDE (P-CDE) in small cell lung cancer (SCLC): preliminary results. Eur Respir J 1993; 6: 128 S.

32. Bodey GP, Buckley M, Sathe YS, Freireich EJ. Quantitative relationships between circulating leukocytes and infection in patients with acute leukemia. Ann Intern Med 1966: 64: 328-340.

33. Lebeau B, Chastang $\mathrm{Cl}$, Charransol A, et al. Risk factors of death due to bone marrow hypoplasia induced by chemotherapy (CT) without CSF in 1,350 patients with small cell lung cancer (SCLC). Lung Cancer 1994; 11: 84.

34. Radford JA, Ryder NDJ, Dodwell D, Anderson H, Thatcher N. Predicting septic complications of chemotherapy: an analysis of 381 patients treated for small cell lung cancer without dose reduction after major sepsis. Eur $J$ Cancer 1993; 29A: 81-86.

35. Hughes WT, Armstrong D, Bodey GP, et al. From the Infectious Diseases Society of America. Guidelines for 
the use of antimicrobial agents in neutropenic patients with unexplained fever. J Infect Dis 1990; 161: 381-396.

36. Bodey GP, Fainstein V, Elting IS, et al. Beta-lactam regimens for the febrile neutropenic patient. Cancer 1990; 65: 9-16.

37. Rolston KV, Berkley P, Bodey GP, et al. A comparison of imipenem to ceftazidime with or without amikacin as empiric therapy in febrile neutropenic patients. Arch Intern Med 1992; 152: 283-291.

38. Fukuoka M, Furuse K, Saijo N, et al. Randomized trial of cyclophosphamide, doxorubicin, and vincristin versus cisplatin, and etoposide versus altemation of these regimens in small cell lung cancer. J Natl Cancer Inst 1991; 83: 855-861.

39. Bunn PA, Cullen M, Fukuoka M, et al. Chemotherapy in small cell lung cancer: a consensus report. Lung Cancer 1989; 5: 127-134.

40. Miles DW, Fogarty O, Ash CM, et al. Received doseintensity: a randomized trial of weekly chemotherapy with and without granulocyte colony-stimulating factor in small-cell lung cancer. J Clin Oncol 1994; 12: 77-82.

41. Hamm JT, Schieller J, Cuffies C, Oken M, Shepherd F, Kaiser G. Dose ranging study of recombinant human granulocyte-macrophage colony stimulating factor in small cell lung carcinoma. J Clin Oncol 1994; 12: 2667-2676.

42. Arriagada R, Le Chevalier T, Pignon JP, et al. Initial chemotherapeutic doses and survival in patients with limited small-cell lung cancer. N Engl J Med 1993; 329: 1848-1852.

43. Corley RS, Suwanjindar P, Johnson JL, Bagby GC. The influence of hematopoietic growth factors (HGF) on the proliferation of bronchogenic cancers cells of the small cell type. Am Rev Respir Dis 1989; 139: A217.

44. Yamashita Y, Nara N, Aoki N. Antiproliferative and differentiative effect of macrophage colony-stimulating factor on a variant human small cell lung cancer cell line. Cancer Res 1989; 49: 5334-5338.

45. EORTC Clonogenic Assay Screening Study Group. Human granulocyte-macrophage colony-stimulating factor modulates in vitro growth in only a minority of continuous human tumour cell lines. Eur J Cancer 1991; 27: 231- 235.

46. Vellenga E, Biesma B, Meyer C, Wagteveld L, Esselink $\mathrm{M}$, de-Vries EG. The effects of five hematopoietic growth factors on human small cell lung carcinoma cell line: interleukin-3 enhances the proliferation in one of the eleven cell lines. Cancer Res 1991; 51: 73-76.

47. Nichols CR, Fox EP, Roth BJ, Williams SD, Loehrer PJ, Einhorn LH. Incidence of neutropenic fever in patients treated with standard-dose combination chemotherapy for small cell lung cancer and the cost impact of treatment with granulocyte colony-stimulating factor. J Clin Oncol 1994; 12: 1245-1250.

48. Eguchi K, Sasaki S, Tamura T. Dose escalation study of recombinant human granulocyte colony-stimulating factor (KRN8601) in patients with advanced malignancy. Cancer Res 1989; 49: 5221-5224.

49. Eguchi K, Shinkai T, Sasaki Y, et al. Subcutaneous administration of recombinant human granulocyte colonystimulating factor (KRN8601) in intensive chemotherapy for patients with advanced lung cancer. Jpn J Cancer Res 1990; 81: 1168-1174.

50. Sheehan RG, Balaban EP, Frenkel EP. The impact of dose intensity of standard chemotherapy regimens in extensive stage small cell lung cancer. Am J Clin Oncol 1993; 16: 250-255.
51. Urban T, Couderc JL, Nouvet G, et al. Cisplatin, cyclophosphamide, doxorubicin, etoposide and filgrastim in small cell lung cancer. Proceedings Firth International Congress Anti-Cancer Chemotherapy, 1995; 0603.

52. O'Donnell MR, Ruckdeschel JC, Baxter D, et al. Intensive induction chemotherapy for small cell anaplastic carcinoma of the lung. Cancer Treat Rep 1985; 69: 571575.

53. Wolff SN, Birch R, Sama P, et al. Randomized doseresponse evaluation of etoposide in small cell carcinoma of the lung: a Southeastern Cancer Study Group Trial. Cancer Treat Rep 1986; 70: 583-587.

54. Ihde D, Johnson BE, Mulshine JL, et al. Randomised trial of high-dose versus standard dose etoposide and cisplatin in extensive disease stage small cell lung cancer (SCLC). Proc Am Soc Clin Oncol 1987; 6: 181.

55. Woll PJ, Hodgetts J, Lomax L, Bildet F, Cour-Chabernaud $\mathrm{V}$, Thatcher N. Can cytotoxic dose-intensity be increased by using granulocyte colony-stimulating factor? A randomized controlled trial of lenograstim in small cell lung cancer. J Clin Oncol 1995; 13: 652-659.

56. Green JA, Clark Pl, Thatcher N, et al. Filgrastim with dose-intensified CDE chemotherapy in small cell lung cancer. Eur J Cancer 1993; 29A: 557.

57. Gridelli M, D'Aprile M, Palmei S, et al. Phase I study of carboplatine, epirubicin and VP-16 plus G-CSF in extensive small cell lung cancer (SCLC). Eur J Cancer 1993; 29A: 5156.

58. Fujitawa J, Kami T, Ariyoshi Y, et al. Dose intensification study of carboplatin (CBDCA) and etoposide (VP16) with G-CSF in small cell lung cancer (SCLC). Proc Am Soc Clin Oncol 1993; 12: 331.

59. Sheridan WP, Begley CG, Juttner CA, et al. Effects of peripheral blood progenitor cells mobilised by filgrastim (G-CSF) on platelet recovery after high-dose chemotherapy. Lancet 1992; 339: 640-644.

60. Pettengell R, Morgenstern GR, Woll PJ, et al. Peripheral blood progenitor cell transplantation in lymphoma and leukemia using single apheresis. Blood 1993; 82: 3770-3777.

61. Langlands K, Craig JO, Parker AC, Anthony RS. Molecular determination of minimal residual disease in peripheral blood stem cell harvests. Bone Marrow Transplantation 1990: 5: 64-65.

62. Pettengell R, Woll PJ, Thatcher N, Dexter TM, Testa NG. Multicyclic, dose-intensive chemotherapy supported by sequential reinfusion of hematopoietic progenitors in whole blood. J Clin oncol 1995; 13: 148-156.

63. Maher BW, Lieschke GJ, Green M, et al. Filgrastim in patients with chemotherapy-induced febrile neutropenia. A double-blind, placebo-controlled trial. Ann Intern Med 1994; 121: 492-501.

64. Biesma B, de Vries EG, Willemse PH, et al. Efficacy and tolerability of recombinant human granulocytemacrophage colony-stimulating factor in patients with chemotherapy-related leukopenia and fever. Eur J Cancer 1990; 26: 932-936.

65. Riikonen P, Saarinen UM, Makipernaa A, et al. rhGM-CSF in the treatment of fever and neutropenia: a doubleblind, placebo-controlled study in children with malignancy. Proc Am Soc Clin Oncol 1993; 12: 441.

66. Mayordomo JI, Rivera F, Diaz-Puente M, et al. Decreasing morbidity and cost of treating febrile neutropenia by adding G-CSF and GM-CSF to standard antibiotic therapy: results of a randomized trial. Proc Am Soc Clin Oncol 1993; 12: 437. 